\title{
Characterization of killer immunoglobulin-like receptor genetics and comprehensive genotyping by pyrosequencing in rhesus macaques
}

\author{
Anna J Moreland ${ }^{1}$, Lisbeth A Guethlein², R Keith Reeves ${ }^{3}$, Karl W Broman ${ }^{4}$, R Paul Johnson ${ }^{3}$, Peter Parham², \\ David $\mathrm{H} \mathrm{O}^{\prime}$ Connor ${ }^{1,5}$ and Benjamin N Bimber ${ }^{*^{*}}$
}

\begin{abstract}
Background: Human killer immunoglobulin-like receptors (KIRs) play a critical role in governing the immune response to neoplastic and infectious disease. Rhesus macaques serve as important animal models for many human diseases in which KIRs are implicated; however, the study of KIR activity in this model is hindered by incomplete characterization of KIR genetics.
\end{abstract}

Results: Here we present a characterization of KIR genetics in rhesus macaques (Macaca mulatta). We conducted a survey of KIRs in this species, identifying 47 novel full-length KIR sequences. Using this expanded sequence library to build upon previous work, we present evidence supporting the existence of 22 Mamu-KIR genes, providing a framework within which to describe macaque KIRs. We also developed a novel pyrosequencing-based technique for KIR genotyping. This method provides both comprehensive KIR genotype and frequency estimates of transcript level, with implications for the study of KIRs in all species.

Conclusions: The results of this study significantly improve our understanding of macaque KIR genetic organization and diversity, with implications for the study of many human diseases that use macaques as a model. The ability to obtain comprehensive KIR genotypes is of basic importance for the study of KIRs, and can easily be adapted to other species. Together these findings both advance the field of macaque KIRs and facilitate future research into the role of KIRs in human disease.

\section{Background}

Killer immunoglobulin-like receptors (KIRs) are a highly polymorphic family of cell surface receptors expressed on natural killer (NK) cells and a subset of T-lymphocytes [1-3]. KIR mediated signaling plays a key role in the identification of foreign cells and the antiviral response [4-9]. The best characterized KIR ligands are major histocompatibility complex class I (MHC-I) molecules, although ligands have not been identified for all KIRs [10,11]. Because both KIRs and MHC-I are highly polymorphic, host genotype plays an important role in KIR function.

KIR genetic diversity can be described in terms of polymorphism and polygenicity. To date, there are 15

\footnotetext{
* Correspondence: bimber@wisc.edu

${ }^{5}$ Wisconsin National Primate Research Center, University of Wisconsin-

Madison, Madison, Wisconsin 53706, USA

Full list of author information is available at the end of the article
}

$K I R$ genes described in humans [12]. The number of KIR genes varies between individuals, with 7-12 genes per haplotype $[13,14]$. Because the protein product of each KIR gene generally binds a unique set of ligands, the subset of KIRs encoded by an individual dictates the potential KIR interactions that can occur. In addition to variation in gene content between haplotypes, there is allelic polymorphism within each KIR gene [13]. Broadly speaking, the allotypic variants encoded by a KIR gene bind the same subset of MHC-I ligands, although exceptions do exist [15]. Distinct KIR allotypes can have differing binding affinities for particular MHC-I allotypes. These differences in KIR/MHC-I binding affinity can alter KIR signaling and NK cell activity [16]. In addition to KIR genotype, $M H C-I$ genotype must be considered since it determines the set of available KIR ligands and since it is possible to express a KIR with or without its cognate MHC-I molecule $[10,17]$. 
Specific $K I R / M H C-I$ genotypes have been implicated as a factor contributing to the immune control of multiple human diseases including hepatitis $C$ virus, human papilloma virus, malaria, and human immunodeficiency virus (HIV) $[6-9,18]$. One of the best-studied examples of $K I R / M H C-I$ genetics and disease is that of KIR3DL1/ KIR3DS1 and HLA-Bw4 in HIV infection. Individuals who express specific KIR3DL1/KIR3DS1 alleles in combination with certain $H L A-B$ alleles containing the Bw4 motif show slower progression to AIDS $[8,9]$. This genetic association has more recently been supported by functional data demonstrating that NK cells expressing KIR3DS1 have increased anti-HIV activity against target cells expressing HLA-Bw4, although the underlying mechanism remains to be elucidated [19].

Despite advances in our understanding of KIR biology, the mechanisms through which specific KIR/MHC-I combinations influence disease progression are not fully understood. This is at least partially due to the complexity of $K I R / M H C-I$ genotypes and difficulty in identifying KIR/MHC-I matched cohorts. Rhesus macaques (Macaca mulatta) are an established and widely used experimental model system for many human diseases, including immunodeficiency virus [20]. The advantages of studying infectious disease in rhesus macaques include the ability to manipulate the dose, route, and strain of the infectious agent, as well as the ability to analyze specimens from defined time points. For the study of KIR activity, perhaps the most important advantage is the ability to select subjects based on genetics. This benefit is evidenced by the work in macaques to elucidate the role of the cytotoxic T-lymphocyte (CTL) response in immunodeficiency viral infection, which is also heavily dependent on host genetics [21,22].

Macaque KIRs have received less study than human KIRs. While previous work shows that macaque KIRs have structure and genomic organization similar to human KIRs, and suggests that they play a similar functional role, these studies also demonstrate that there has been considerable evolution within macaque KIRs since the species diverged [23]. While 15 genes have been described in humans, the number and identity of the KIR genes present in macaques is distinct. Developing an understanding of the KIR genes present in this species and an overall assessment of KIR genetic diversity is a matter of practical importance for the use of macaques as a model for KIR function. Using cDNA sequences, an initial model for macaque $K I R$ genetic organization was formed containing 18 putative KIR groups $[23,24]$. In addition to the sequence of KIR transcripts, the genomic sequence of one rhesus macaque KIR haplotype has been described [25]. More recent studies have added to the total number of described macaque $K I R$ sequences [23,24,26-28]. With more sequence data available, phylogenetic relationships became clearer, and the model of macaque KIR genetics has been refined. This body of work has been used to create a model for macaque KIR genetic organization and to develop a formal system of nomenclature (Guethlein et al, in preparation).

Here we present the results of a survey of rhesus macaque $K I R$ genetics. Using full-length cloning, we identified 47 novel full-length rhesus macaque KIRs, substantially increasing the library of known sequences. Using this expanded library, we performed phylogenetic analysis supporting the existence of 22 rhesus macaque KIR genes. Together with previously published KIR sequences, this provides a framework with which to describe $K I R$ genetics in this species. In addition to improving our understanding of macaque KIRs at the population level, we developed a novel pyrosequencingbased approach for KIR genotyping. This technique provides both comprehensive KIR genotyping and frequency estimates for expression of each $K I R$ transcript. The findings presented here, along with the novel techniques set forth, should serve as a foundation for further research on rhesus $K I R$ genetics and for defining KIR function in this important animal model.

\section{Methods}

\section{Animal Care and Specimen Processing}

Animals used in this study were housed and cared for by the trained veterinary staff at the Wisconsin National Primate Research Center (WNPRC) or the New England Primate Research Center (NEPRC). All procedures were approved by the host institution's Animal Care and Use Committee. Nucleic acid was obtained from peripheral blood mononuclear cells (PBMC) or purified natural killer (NK) cells, as indicated. RNA purification was accomplished using either the MagnaPure LC Total Nucleic Acid Purification kit (Roche, Branford, CT) or the DNA/RNA Allprep Kit (QIAGEN, Valencia, CA) according to the manufacturer's instructions.

NK cells were isolated from whole PBMC by negative magnetic bead fractionation. First, PBMC were incubated for 20 minutes at room temperature in $0.1 \% \mathrm{BSA} /$ PBS with a cocktail of cross-reactive human monoclonal IgG antibodies composed of the following: anti-CD3 (clone SP34-2, BD Biosciences, La Jolla, CA), anti-CD14 (clone M5E2, BD Biosciences), anti-CD40 (clone 5C3, BD Biosciences), and anti-CD66/CEACAM (clone TET2, AbCam, Cambridge, MA or Santa Cruz Biotechnology, Santa Cruz, CA). Next, antibody-coated PBMC were washed and resuspended in $0.1 \%$ BSA/PBS then incubated for 35 minutes at room temperature with Pan-IgG Dynabeads (Dynal Biotech, Norway) at a 4:1 bead-to-cell ratio. The suspension was then placed on a Dynal magnet and the unbound cells were collected. 
Purity was assessed by flow cytometry with $>90 \%$ of collected cells routinely bearing an NK cell phenotype of

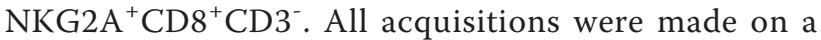
FACSCalibur (BD Biosciences) and analyzed using FlowJo software (Tree Star Inc., Ashland, OR).

\section{Full-Length cDNA Cloning and Sequencing}

First-strand cDNA was synthesized using the Superscript III First-Strand One-Step RT-PCR kit (Invitrogen, Carlsbad, CA) according to the manufacturer's instructions. PCR amplification was performed using Phusion high-fidelity polymerase (New England Biolabs, Ipswich, MA) and the following external primers: 5'-CAGCACCATGTCGCTCAT-3' and 5'-GGGGTCAAGTGAAGTGGAGA-3'. PCR conditions were: $98^{\circ} \mathrm{C}$ for $30 \mathrm{~s}, 28$ cycles of $98^{\circ} \mathrm{C}$ for $5 \mathrm{~s}, 63^{\circ} \mathrm{C}$ for $1 \mathrm{~s}, 72^{\circ} \mathrm{C}$ for $20 \mathrm{~s}$, and a final extension at $72^{\circ} \mathrm{C}$ for $5 \mathrm{~min}$. PCR products were cloned into pCR-Blunt TOPO (Invitrogen, Carlsbad, CA) and bidirectionally sequenced using DYEnamic ET Terminator cycle sequencing kit (GE Healthcare, Piscataway, NJ). Internal primers used in sequencing were $5^{\prime}$ AACCTTCCCTCCTGGCC-3 and 5'-TTGGTTCAGT GGGTGAAGGCCAA-3.' CodonCode Aligner (CodonCode Corporation, Dedham, MA) was used for sequence analysis, and in order to minimize error introduced by PCR artifacts, novel alleles were only included when three or more identical full-length cDNA clones were observed. Novel sequences have been deposited in Genbank (Additional file 1, Table S1). Novel full-length sequences were assigned formal names through the Immuno Polymorphism Database [29].

\section{Phylogenetic Analysis}

The cDNA sequences obtained in this analysis and from previous studies $[23,25,27,28,30]$ were aligned using Clustal X [31] and manually corrected in BioEdit (Ibis Therapeutics, Carlsbad, CA). Phylogenetic trees for the complete dataset were made using both neighbor-joining (1000 replicates, pairwise deletion, Tamura-Nei, in MEGA4) and parsimony (1000 replicates in Paup). These trees were used to divide the sequences into Mamu-KIR3DL20, -KIR2DL04, -KIR1D, and -KIR3D (lineage II) groups. Subsequent analysis was restricted to the lineage II dataset using the same methods. Alleles of Mamu-KIR3DL11, -KIR3DS01, -KIR3DS03, -KIR3DSW07, and $-K I R 3 D S W 08$ were not obtained in this study and sequences for these genes were taken from GenBank $[23,27,30,32]$.

A dataset containing consensus sequences for each of the lineage II KIR genes was constructed using all known sequences to compute the consensus sequence. For groups containing only two allotypes, one of the allotypes was chosen to represent the consensus. The sequences used are indicated in Additional file 1, Figure S1. The individual extracellular Ig domains (D0, D1, and D2) were analyzed separately using the methods described above. The stem, transmembrane and cytoplasmic tails were not included in this analysis as previous reports have shown that the stem, transmembrane, and tail of macaque activating KIRs share similarity to KIR2DL4 and are distinct [23].

\section{PCR Amplification and Pyrosequencing}

As with our full-length products, cDNA was synthesized using the Superscript III First-Strand One-Step RT-PCR kit (Invitrogen, Carlsbad, CA). cDNA-PCR amplicons spanning 623 base pairs of the D1 and D2 domains were synthesized using Phusion high-fidelity polymerase (New England Biolabs, Ipswich, MA). Each PCR primer contained a target-specific sequence, an MID tag, and an adapter sequence (Additional file 1, Table S2). PCR conditions were: $98^{\circ} \mathrm{C}$ for $30 \mathrm{~s}, 28-33$ cycles of $98^{\circ} \mathrm{C}$ for $5 \mathrm{~s}, 61^{\circ} \mathrm{C}$ for $1 \mathrm{~s}, 72^{\circ} \mathrm{C}$ for $20 \mathrm{~s}$, and a final extension at $72^{\circ} \mathrm{C}$ for 5 min. cDNA-PCR product purification was accomplished using Ampure XP beads (Agencourt, Beverly, MA) according to the manufacturer's instructions. Amplicons were then normalized to equimolar concentrations and grouped into pools of twelve samples for Titanium amplicon pyrosequencing. Emulsion PCR, Roche/454 Titanium amplicon pyrosequencing, image processing, and base calling were performed according to the manufacturer's instructions (Roche/454 Life Sciences, Branford, CT) at the University of Illinois at Urbana-Champaign High-Throughput Sequencing Center. Each pool of twelve samples was sequenced in onesixteenth of a $70 \times 75$ PicoTiterPlate.

\section{Sequence Analysis}

Pyrosequencing flowgram data was processed using a custom analysis pipeline. Briefly, data were trimmed by sequence quality and aligned against a reference database of all known macaque $K I R$ sequences using the Mosaik aligner (http://code.google.com/p/mosaikaligner/). The reference library of $K I R$ sequences was obtained from the Immuno Polymorphism Database [29]. Polymorphisms between reads and the reference sequences were scored with custom scripts that utilized Samtools and BioPerl [33,34]. The source code for this pipeline can be obtained from a subversion repository (https://hedgehog.fhcrc.org/tor/stedi/trunk/ server/customModules/SequenceAnalysis). The pipeline itself has been integrated into the LabKey Software platform as the SequenceAnalysis module, which provides a graphical, web-based platform to initiate analysis pipelines and view results. LabKey is a free, open source software package available at http://www. labkey.org. Sanger sequence data was analyzed using CodonCode Aligner (CodonCode Corporation, Dedham, MA). 
In order to reduce errors introduced by PCR artifacts, $K I R$ sequences were only considered to be present in an animal if they represented one percent or more of total $K I R$ sequence reads from that animal. In order to identify novel alleles not present in the reference library, unaligned sequences were then assembled in CodonCode Aligner at $100 \%$ identity. BLAST analysis was performed for the resulting contigs against a database of published Mamu-KIR sequences. Unaligned sequences were deemed novel when they represented at least one percent of total sequence reads from at least one animal and did not represent a potential insertion/deletion error in the pyrosequencing base-calling.

\section{Results}

Rhesus macaque KIR diversity

$K I R$ genotype has a significant influence on NK cell activity. Studying the role of macaque $K I R$ genetics in disease pathogenesis first requires an understanding of total diversity within the population and a framework within which to describe it. To date, 149 distinct fulllength Mamu-KIR sequences have been deposited in Genbank. By comparison, there are more than 615 distinct $K I R$ alleles identified in humans-more than four times as many alleles as have been identified to date in rhesus macaques [35]. Thus, the previously catalogued alleles likely represent only a fraction of total Mamu$K I R$ diversity. In order to expand the repertoire of known Mamu-KIR alleles, we performed full-length cloning and sequencing of $K I R$ alleles from 17 Indian rhesus macaques. These animals yielded 47 novel $K I R$ alleles, a substantial expansion of documented rhesus macaque KIR diversity (Additional file 1, Table S1). The novel sequences have been assigned names following the KIR genes and nomenclature conventions established in the Non-human Primate KIR Nomenclature Report (Guethlein et al, in preparation) ${ }^{1}$.

As a first step in the analysis of the rhesus macaque sequences recovered from cloning, phylogenetic trees based on the full-length sequences were constructed. Human KIRs have been divided by phylogenetic analyses into four lineages ${ }^{2}$. Macaque KIRs assorted into groups matching these lineages. The sequences assorted into Mamu-KIR2DLO4 (lineage I), -KIR3D (lineage II), -KIR1D (lineage III), and -KIR3DL20 (lineage V), with the majority of sequences resembling human lineage II, in accordance with previous publications [25,30,36]. Relatively little allelic variation was observed within the Mamu-KIR2DL04, -KIR3DL20, and -KIR1D groups (data not shown). In contrast, the Mamu-KIR3D lineage II sequences were highly diverse. Our sequences assorted into 19 distinct groups corresponding to 19 of the rhesus macaque lineage II genes (Figure 1 and Additional file 1, Figure S1). An important point to note is that rather than increasing the number of detected $K I R$ genes, most novel sequences share strong similarity with established macaque KIR genes. Therefore, while there are likely a large number of undiscovered macaque $K I R$ alleles, the most common macaque $K I R$ genes may now have been identified.

There is evidence for extensive recombination among the Mamu-KIR sequences described here. To identify recombination events affecting individual alleles, sequences were analyzed with the RDP (recombinant detection program) package and inspected manually (data not shown). In addition, domain-by-domain phylogenetic analysis using consensus sequences for each gene revealed extensive domain sharing between macaque $K I R$ genes (Figure 2). This suggests that the genes are themselves products of ancient duplication and recombination events. This mechanism for the generation of $K I R$ genes is consistent with observations of KIRs in other species [37]. Pairs of KIRs with similar extracellular domains but differing cytoplasmic tails have been observed in other species, with human KIR3DL1/3DS1 being a notable example [38]. In this analysis we also found such pairing, with the bestmatched pair being Mamu-KIR3DLW03 and MamuKIR3DS05. Other pairs were identified that were similar in two of the three extracellular Ig-like domains, for example Mamu-KIR3DLO7 and Mamu-KIR3DSW09. Cytoplasmic domains were not included in this analysis, although it should be noted that macaque long and short cytoplasmic tails are phylogenetically distinct, with the latter resembling the tail of human KIR2DL4 [23].

\section{Comprehensive KIR genotyping by pyrosequencing}

The expanded library of macaque KIR sequences described in this publication and others has enabled the creation of a framework to describe macaque KIRs, and its phylogenetic analysis suggests that the most common KIR genes have been identified [24-28,30,32]. For the study of KIRs in disease pathogenesis, one of the most basic requirements is the ability to identify the KIRs expressed by individual subjects. KIRs pose many challenges for genotyping: each subject expresses a distinct number of KIRs, there is extensive sequence homology between KIR transcripts, splice variants may be functionally significant, and pseudogenes are common. The cloning-based strategy we employed for allele discovery can be used to provide genotypes of individual subjects; however, this method is labor intensive and will frequently miss low-abundance transcripts due to the limited number of clones examined. Techniques such as sequence-specific PCR (SSP) can be used to identify the presence or absence of a particular gene or allele; however, sequence homology between KIRs complicates primer design, and a large number of primer pairs would be required for comprehensive genotyping. 


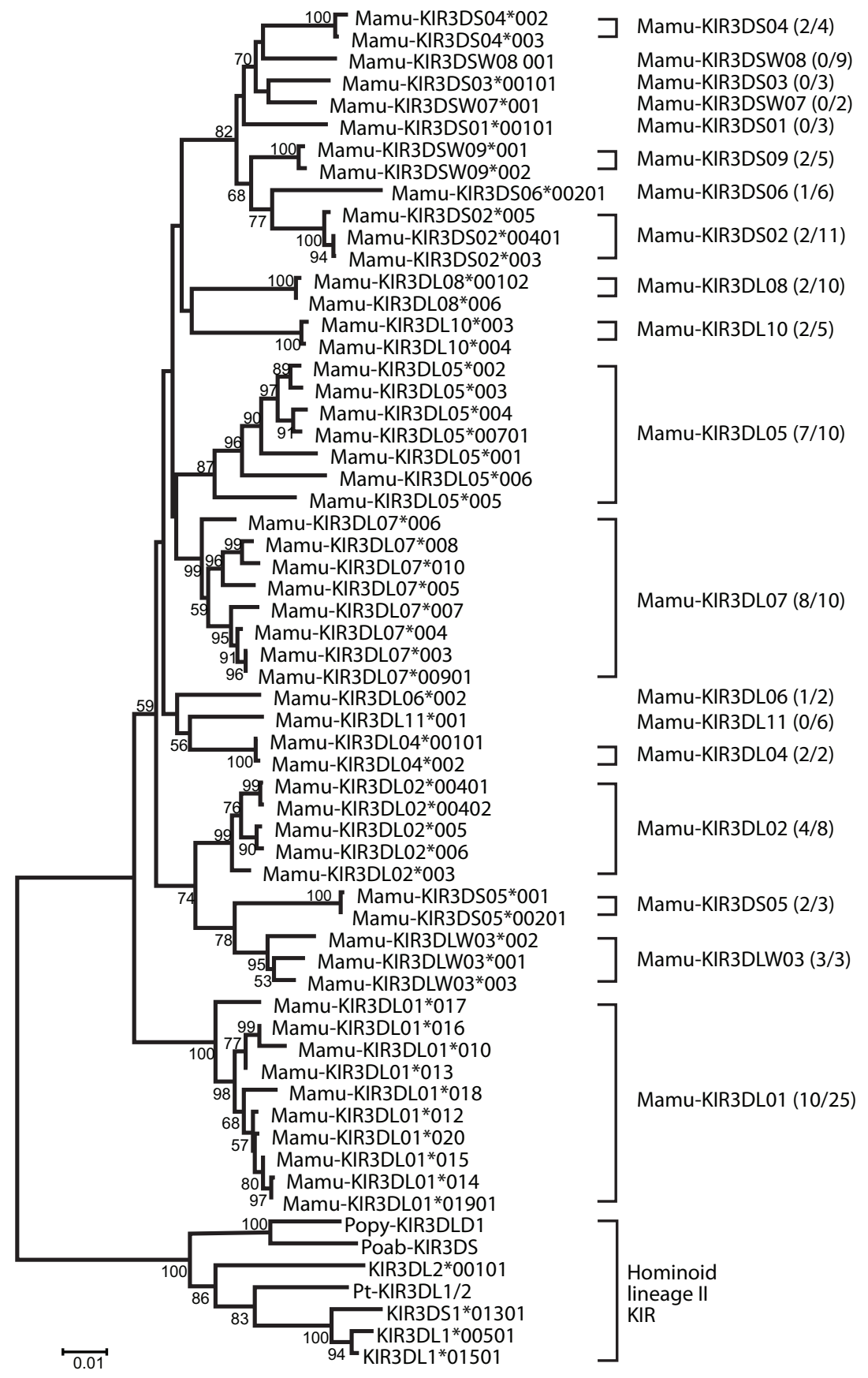

Figure 1 Rhesus macaque lineage II KIR alleles form 19 distinct groups. Neighbor-joining and parsimony analysis was performed using predicted amino-acid sequences for the dataset comprising the lineage II sequences obtained in this study. The neighbor-joining tree is shown, which was not significantly different from the parsimony tree [data not shown]. Bootstrap support of greater than $50 \%$ is indicated. Representative hominoid lineage II KIR were used as an outgroup. For each gene, the numbers in parentheses indicate the total number of alleles discovered in this study compared with the total number known. 


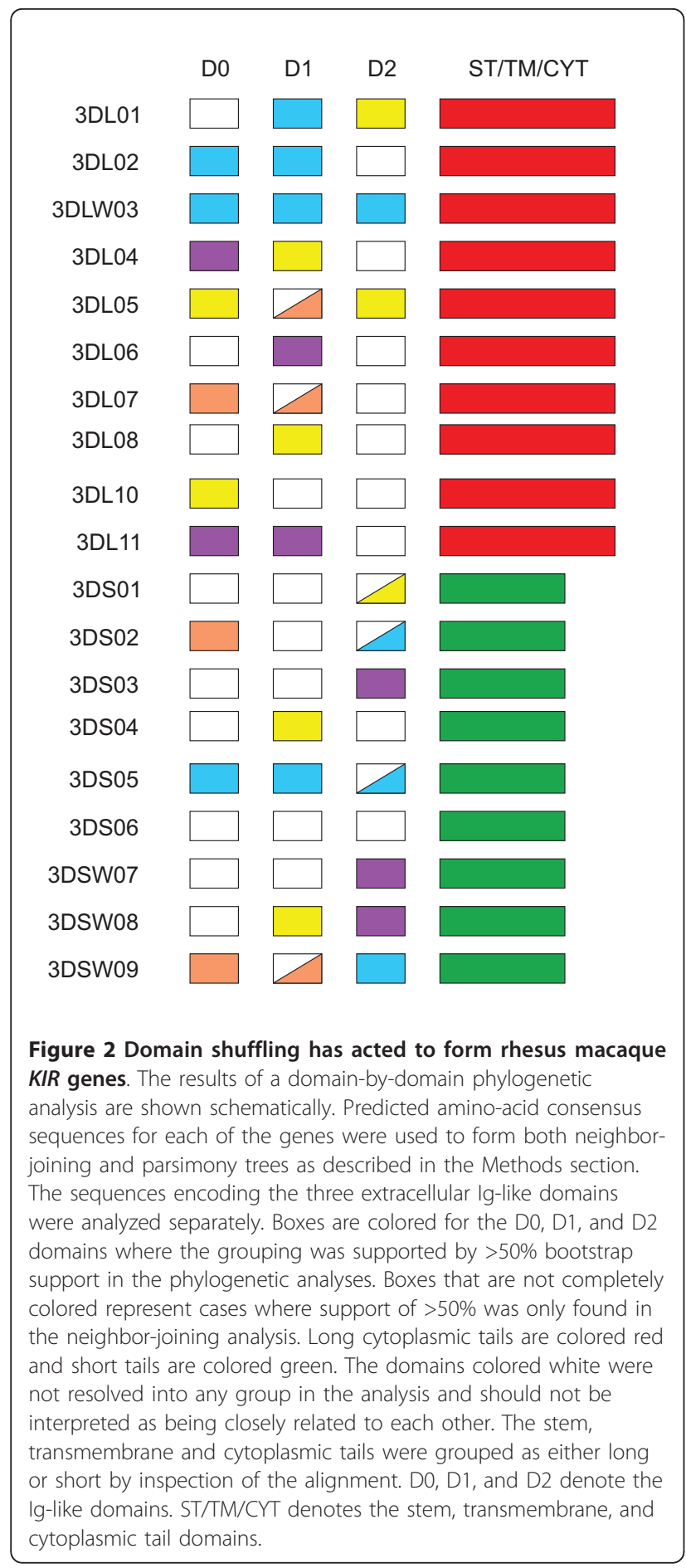

To overcome these limitations, we developed a novel sequence-based typing approach. Recent developments in Roche/454 Titanium pyrosequencing technology have enabled sequencing of cDNA-PCR amplicons with high sensitivity and throughput. To adapt this approach for $K I R$ genotyping, we first identified primer sites that are highly conserved across all published Mamu-KIR3D sequences (Figure 3). These primers amplify a region of 623 bp spanning the majority of D1, all of D2, and part of the stem region. This amplicon was selected to span a polymorphic region of the transcript and to maximize conservation of primers. These primer sites are also conserved amongst most published МатuKIR1D alleles-although they will not amplify MamuKIR2DL04 sequences, as these lack the D1 region and therefore the 5 ' primer-binding site. These primers were used for PCR on CDNA derived from total RNA, producing amplicons representing the distinct MamuKIR3D and -KIR1D transcripts expressed by each subject. Pyrosequencing of these amplicons produces clonal sequence reads corresponding to individual input transcripts. Collectively, these reads represent the KIRs expressed by the subject, with the exception of MamuKIR2DL04. A schematic of this process is shown in Figure 4.

Using this approach, we genotyped PBMC samples from 61 animals. We detected an average of 1836 total sequence reads per animal, representing an average of 10.7 distinct transcripts per animal. The KIR genotypes of three half-siblings are shown in Figure 5. For each animal, the distinct KIRs detected are shown, along with the relative frequency of each KIR. In most cases, each sequence read unambiguously matched a single KIR allele, providing typing resolution to the allele level. When reads were identical to more than one known $K I R$, the result is presented either as a set of alleles (ie.

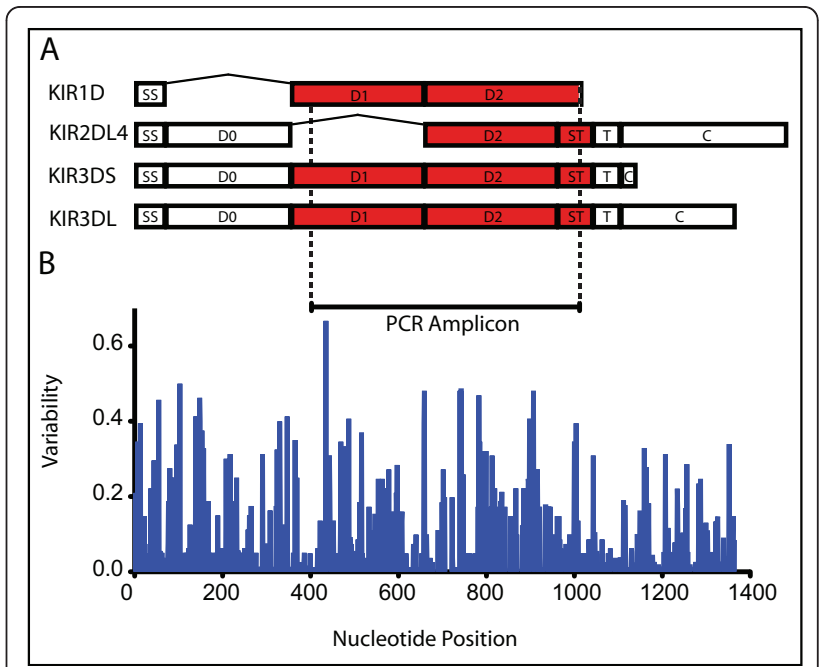

Figure 3 KIR 454 Titanium Amplicon Primer Design. A) Schematic representation of macaque KIR3D and KIR2D molecules showing domain structure. B) Variability plot for a CDNA sequence alignment of all published KIR3DL alleles. PCR primer sites are indicated along with the region amplified by PCR. Note that 454 sequencing reads span the D1, D2, and stem (ST) regions. The signal sequence (SS), D0, transmembrane region (T), and cytoplasmic region (C) are not amplified by our PCR primers. 


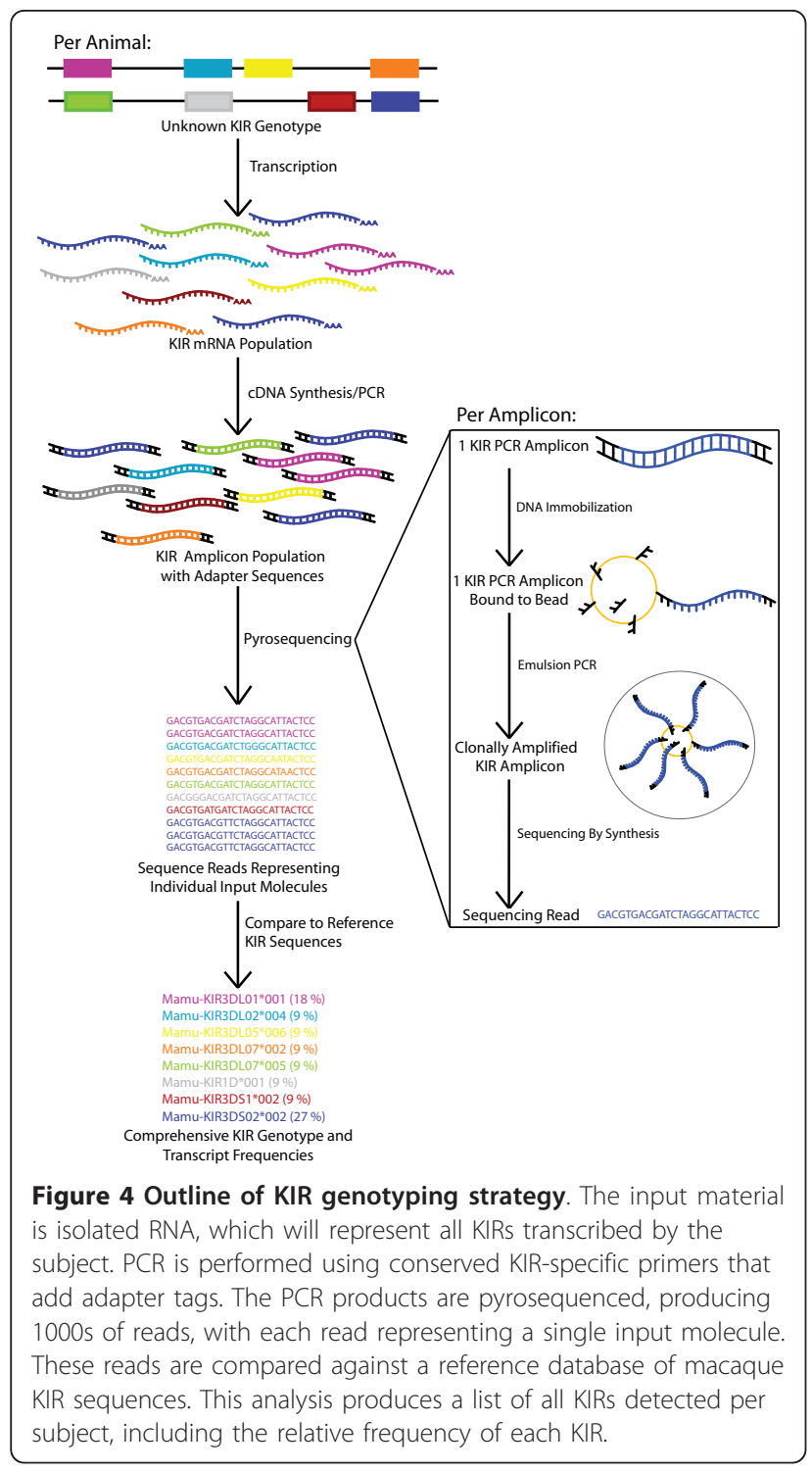

Mamu-KIR3DL10*001/004, meaning the animal is positive for either Mamu-KIR3DL10*001 or "004) or as positive for a gene (ie. Mamu-KIR1Dg, meaning the animal has an allele of this gene). The latter is comparable to the level of resolution commonly provided by sequence specific PCR. To validate this technique, we compared the results of pyrosequencing with cloning data (Figure 5 and Additional file 1, Table S3). Because pyrosequencing examines hundreds or thousands of clones, as opposed to the tens of clones generally examined in conventional cloning, the former is able to detect more transcripts per animal, providing a more comprehensive genotype. An example is seen in r95061 with MamuKIR3DS06, which was detected by pyrosequencing, but was not detected by cloning. While the 623bp amplicon used in pyrosequencing was frequently able to provide

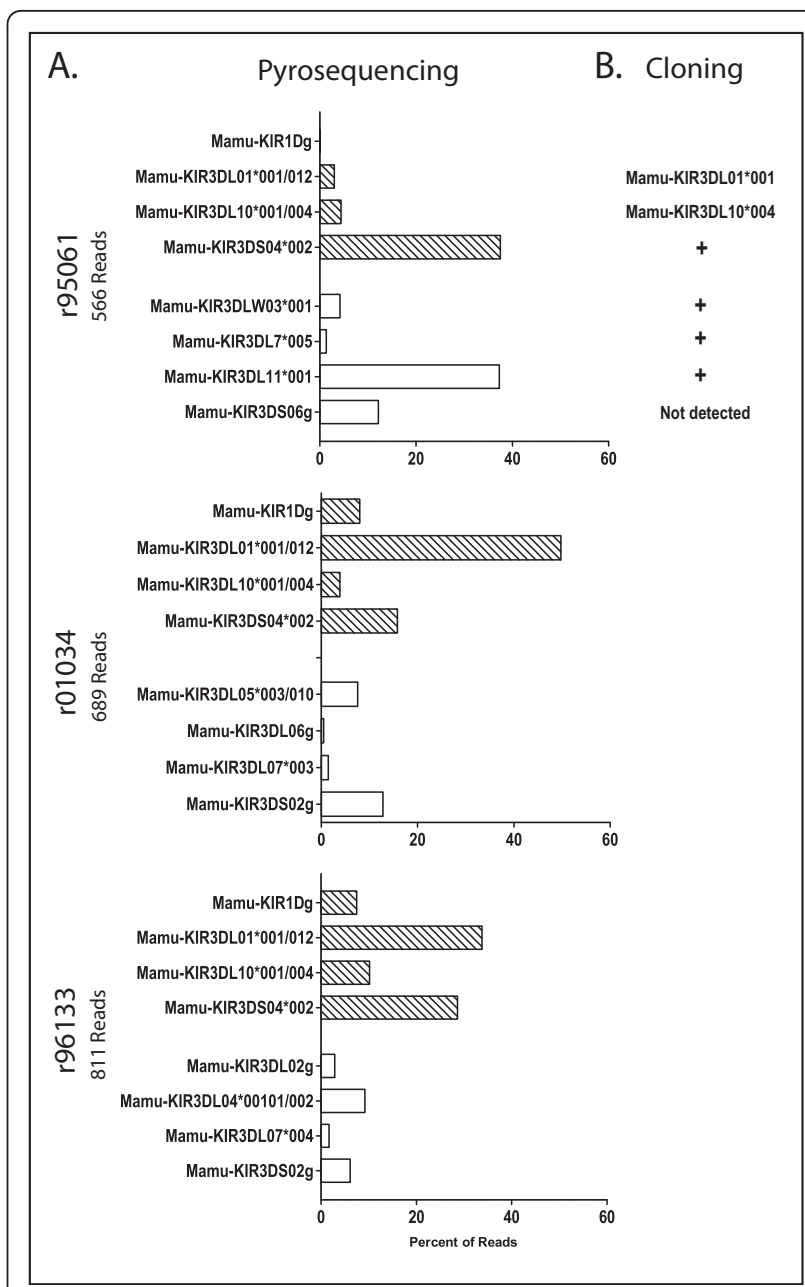

Figure 5 Differential KIR expression between subjects with a common KIR haplotype. A) KIR genotypes obtained by pyrosequencing are shown for three rhesus macaque half-siblings. The relative expression level of each detected $K I R$ allele is shown. Striped bars indicate alleles present on their shared KIR haplotype. The number of pyrosequencing reads is shown to the left of each graph. B) For r95061, the KIR alleles detected by CDNA cloning are shown. A total of 101 clones were examined. If indicated, the allele detected is listed. A plus sign indicates the genotyping resolution obtained by cloning is identical to the resolution obtained by pyrosequencing.

allele-level typing, there are examples for which fulllength cloning provided higher resolution typing, as we observe for KIR3DL01*001 and KIR3DL10*004 in r95061 (Figure 5).

A key advantage of pyrosequencing is that it provides estimates of the relative transcript frequency for each KIR. This is a dimension not usually captured by genotyping techniques. The three animals in Figure 5 share a KIR haplotype containing Mamu-KIR1D, Mamu-KIR3DL1"001, Mamu-KIR3DL10*004 and Mamu-KIR3DSO4*002 (Figure 5, striped bars). KIR expression is influenced by the complete $K I R$ and $M H C$ genotype of the subject $[10,17,39]$. 
Work in humans has shown that $K I R$ genotype is only loosely predictive of KIR RNA expression [40]. While the animals in Figure 5 share one KIR haplotype, their second $K I R$ haplotypes are distinct, and none are $M H C$ identical (data not shown). We observe considerable differences in the expression level of the KIRs on the shared haplotype, which is likely attributable to differences in $K I R / M H C$ genotype. While these findings are not unexpected given the complex regulation of KIR expression, they do underscore the need to examine factors beyond the simple presence or absence of a given KIR.

To validate this technique, we examined the reproducibility of KIR expression levels. We performed two independent NK cell preparations from four animals, and two independent PCRs from each cell preparation. The resulting PCR amplicons were pyrosequenced. A graph of the relative expression level of each $K I R$ is shown in Figure 6, with the full data in Additional file 1, Table S4. The frequency of each KIR was highly reproducible between sample preparations and PCR replicates. In every instance for which the average expression of a $K I R$ was greater than $3 \%$ of total reads, that $K I R$ transcript was detected in all replicates, demonstrating high sensitivity. It should be noted that transcripts present in less than 3\% of total transcripts were missed in some reactions. There was also greater variability in detected expression levels among lower frequency transcripts. Of interest, in three of four animals, a single KIR transcript was dominant, representing greater than $60 \%$ of reads. Animal 225-97 was an exception, where four distinct KIRs comprise between $15-20 \%$ of the population each.

Another advantage of sequence-based typing, as opposed to techniques such as sequence-specific PCR, is that it is not limited to previously described alleles. From this data we were able to characterize an additional 44 novel partial-length rhesus macaque $K I R$ sequences, 32 of which were found in multiple animals. As was the case with our novel full-length sequences, most novel KIR sequences showed significant sequence homology to the phylogenetic groups defined in Figure 1 (Additional file 1, Table S5), further supporting these putative gene groupings. While the majority of novel sequences were similar to these groups, we also identified two novel sequences, KIRnov03 and KIRnov04, that are similar to Mamu-KIR3DSO3, but with a distinctive sequence motif in D1 (Figure 7). These sequences are of interest because they share this motif with a sequence previously found only in cynomolgus macaques (Macaca fascicularis) (EU419113). The unique residues for these sequences (186-193) correspond to predicted MHC class I binding sites [41]. These sequences may represent an additional macaque KIR gene, or a lineage within KIR3DSO3.

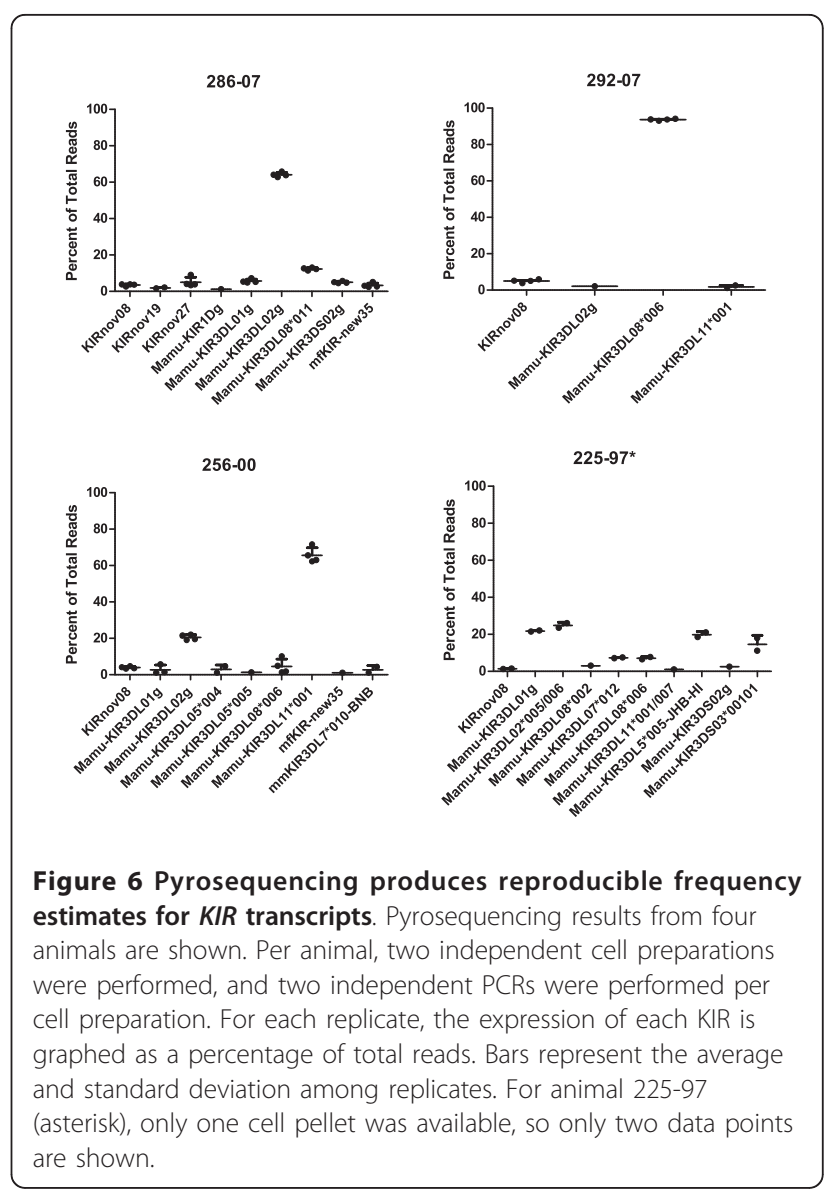

\section{Population survey of KIR gene frequency}

Obtaining comprehensive KIR genotypes from this large cohort allowed us to examine the relative frequency of each $K I R$ gene at the population level (Figure 8A). We combined the pyrosequencing data with full-length cloning data to form a 69-animal cohort. While no KIR gene was present in every animal, Mamu-KIR3DL01 was present in approximately $84 \%$ of the cohort, making it the closest approximation to a framework gene in this population. While Mamu-KIR3DL20, -KIR3DL11, and -KIR3DSW08 have been proposed as framework genes for this species, each was present in less than $25 \%$ of this cohort [27]. It should be noted that our approach will identify transcribed KIRs only, while Kruse et al. employed sequence-specific PCR from genomic DNA. Our previous work in cynomolgus macaques suggests that Mafa-KIR3DL20 is commonly found as a pseudogene [28], which would be detected from gDNA, but not mRNA. It is possible some rhesus macaque haplotypes also have Mamu-KIR3DL20 as a pseudogene. While differences in technique may explain some discrepancies, it is also possible that the distinct breeding populations of rhesus macaques have distinctive genetic compositions. Mamu- KIR2DLO4 has also been 


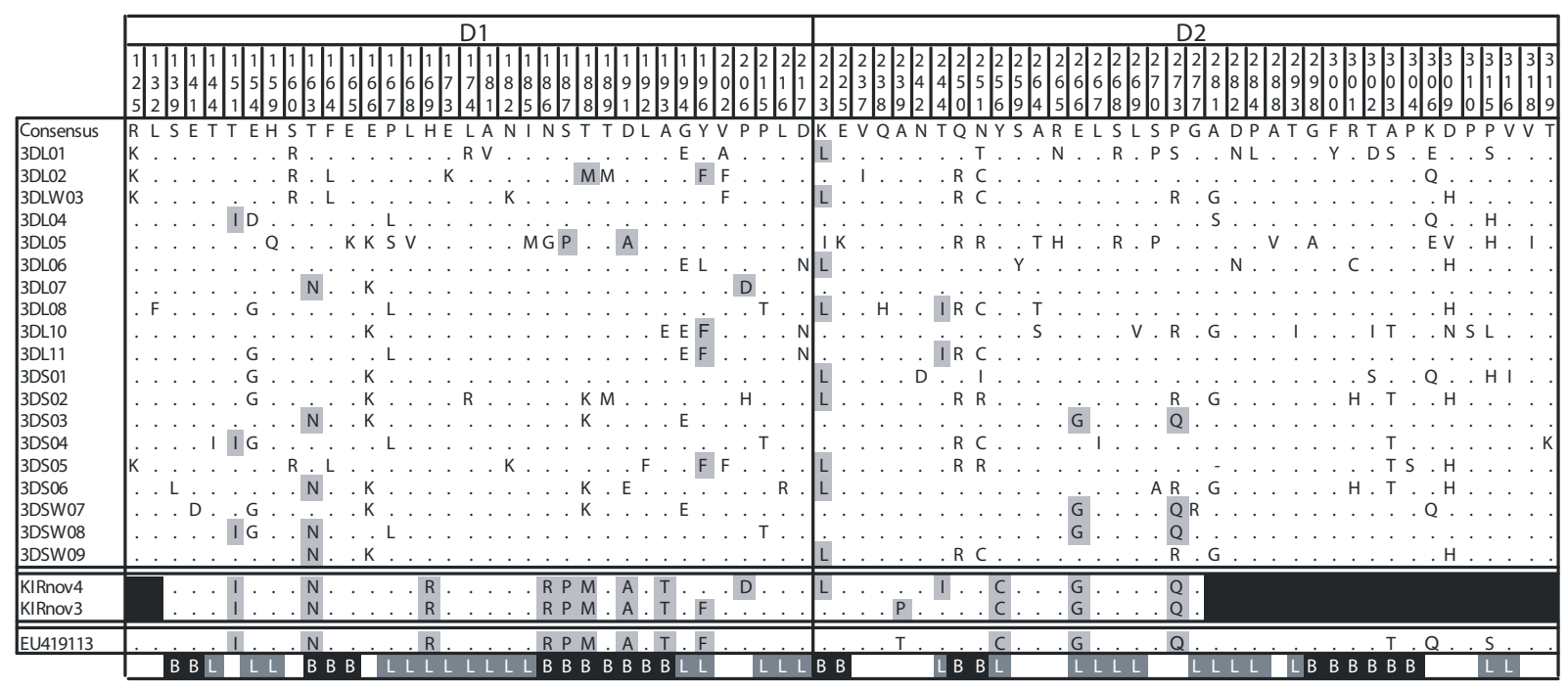

Figure 7 Novel rhesus macaque KIR sequences share homology with cynomolgus macaque KIRs. Amino acid differences between the consensus sequences for the lineage II Mamu-KIR genes, KIRnov03, KIRnov04 and the Mafa-KIR sequence EU419113 are shown. The amino-acid position is indicated above the amino-acid sequences. The positions in the novel sequences that vary from the consensus are highlighted. Residues predicted to be involved in MHC-binding or alpha-helix loops are indicated below the sequences with $B$ or $L$ respectively. Black shading indicates residues for which no sequence coverage was available.

suggested as a framework gene in macaques [27], although -KIR2DLO4 was only identified in 2 of 8 published cynomolgus macaque haplotypes [28]. As noted previously, the primers used in this study select against Mamu-KIR2DLO4 transcripts, so we could not determine the presence or absence of this gene.

Using the pyrosequencing data generated from our cohort of 61 animals, we compared the relative contribution of each KIR gene to the total KIR transcripts identified in each animal (Figure 8B). Although pyrosequencing provides information about the KIR allele, genotyping results were condensed to the gene level for this comparison. The error bars representing the mean and SEM for each gene demonstrate highly variable expression levels for most genes between subjects, with a few KIRs consistently expressed at either high (i.e. Mamu-KIR3DLO8) or low (i.e. Mamu-KIR3DSw09) levels. As observed in Figure 5 , the expression level of a given KIR gene can differ even between genetically similar subjects and is likely influenced by the complete KIR/MHC genotype of that subject. While it would be interesting to examine the relative expression of each KIR in the context of $M H C$ genotype, macaques can express up to 20 distinct $M H C$ class I transcripts and only a handful of macaque KIR/MHC binding partners have been identified $[42,43]$. Therefore this analysis is not practical until physiological macaque KIR/MHC interactions are better understood.

\section{Rhesus macaque KIR haplotypes}

While not all members of this cohort had pedigree information available, there were five distinct pedigreed families within the cohort for which segregation analysis was possible, with a total of 46 animals. By performing segregation analysis using genotypes obtained from cloning and pyrosequencing data, we were able to infer the KIR gene content of 9 haplotypes (Figure 9). Because these haplotypes were defined using segregation analyses, the linear order of KIR genes cannot be conclusively determined and the physical map of gene order shown in Figure 9 is arbitrary. It should be noted that this analysis allows only for definition of the minimum number of genes on a given haplotype, as heterozygosity versus homozygosity is sometimes not possible to rigorously determine, and low-level transcripts may not be detected in all animals with a given haplotype [30]. Among our 9 haplotypes, each was unique as compared to previously published rhesus macaque KIR haplotypes, with an average of 4.6 genes present per haplotype (Range: 3-7) [25,27,28,30]. In accordance with previous studies, we noted one incidence of a duplicated KIR3DL gene (Mamu-KIR3DL10) [27,30]. As described above, there is a notable absence of framework genes among these haplotypes. Mamu-KIR3DLO1 represents the most common KIR in our cohort, yet it is present in only 7 of the 9 haplotypes. 


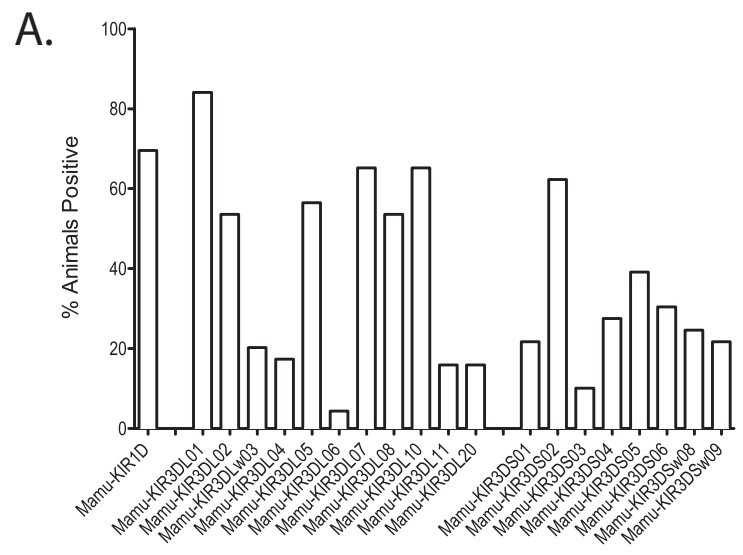

B.

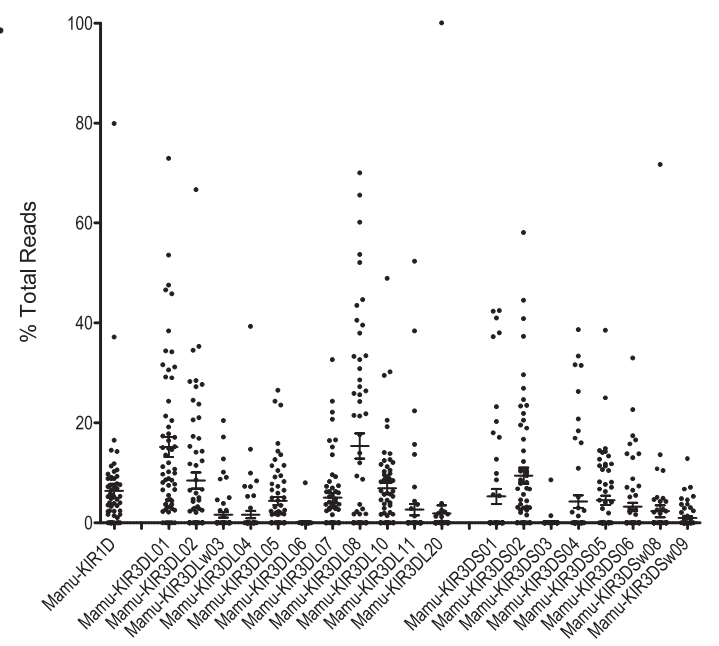

Figure 8 Frequency and relative expression of $K I R$ genes in the rhesus macaque cohort. A) $Y$-axis indicates the percentage of animals within the cohort $(n=69)$ that express the indicated KIR gene. Genes not listed were not present in any animal within our cohort. Mamu-KIR2DL04 was excluded since it is not amplified by our pyrosequencing amplicon. B) Graph illustrates the percent of total pyrosequencing reads per animal $(n=61)$ for each KIR gene. Averages and SEM are represented by error bars. Each animal included had at least 100 sequencing reads. Genotyping results representing less than $1 \%$ of total reads in an animal were excluded to mitigate the influence of PCR artifacts. Ambiguous reads matching more than one KIR gene and splice variants were also excluded.

\section{Discussion}

Killer immunoglobulin-like receptor signaling is implicated in the immune response to numerous human pathogens, yet elucidating the role of KIR mediated signaling in disease pathogenesis is difficult in human subjects. The results of this study advance our understanding of macaque KIRs, enabling the study of KIR activity in this important non-human primate model for many human diseases. This study identified 47 novel full-length rhesus macaque KIRs, a substantial increase in the number of published sequences. Combined with previously published data, our results confirm the existence of 22 common macaque KIR genes, with extensive allelic variation within each gene (Guethlein et al, in preparation). This model of macaque KIR genetics provides an essential framework within which to describe and characterize macaque KIRs. In addition, among the novel sequences found using pyrosequencing, two sequences provide evidence for the existence of either a divergent lineage of Mamu-KIR3DSO3 or an additional gene. While no KIR gene was present in all subjects, Mamu-KIR3DL01 was expressed in $84 \%$ of subjects and 8 genes were present in $>50 \%$ of the cohort. This frequency information may be useful to prioritize KIRs for further functional characterization or the design of cohorts. The comparative lack of expressed framework genes is a distinction from human $K I R$ haplotypes, which may be related to differences in $M H C-I$ genetics. Humans express a maximum of six distinct HLA molecules, from 3 genomic loci. Macaques lack $M H C-I C$, but can express as many as 20 distinct $M H C-I A / B$ alleles [44]. The expansion of macaque lineage II KIR3D loci, with a larger number of loci and few dominant genes, likely mirrors the expansion of macaque $M H C-I A / B$.

We also present a novel pyrosequencing-based approach for KIR genotyping. While this technique was developed in macaques, it could easily be applied to other species, including humans. The ability to characterize the KIR genotype of individual subjects is a basic requirement for the study of KIR function; however, the KIR region presents many challenges for genotyping. The approach we present comprehensively identifies the expressed KIR transcripts and provides a semi-quantitative measure of the relative expression level for each KIR. Our data demonstrate that even subjects sharing a $K I R$ haplotype can have widely different expression levels of these shared KIRs. NK cells undergo complex regulation of KIR expression [10,17]. NK clones expressing fewer distinct KIRs can have a reduced activation threshold under certain conditions, resulting in enhanced NK cell activity [45]. The relative expression of each KIR therefore provides potentially important functional information.

While this and other recent studies have advanced our understanding of the genetic organization and diversity of macaque KIRs, KIR activity must be understood in the context of $M H C$ genotype. The limited knowledge of functional KIR/MHC binding partners remains a key obstacle. Recent work has identified multiple macaque KIR/MHC-I interactions, including allotypes of MamuKIR3DL05, -KIR3DLW03, -KIR3DL11 and -KIR3DS05 


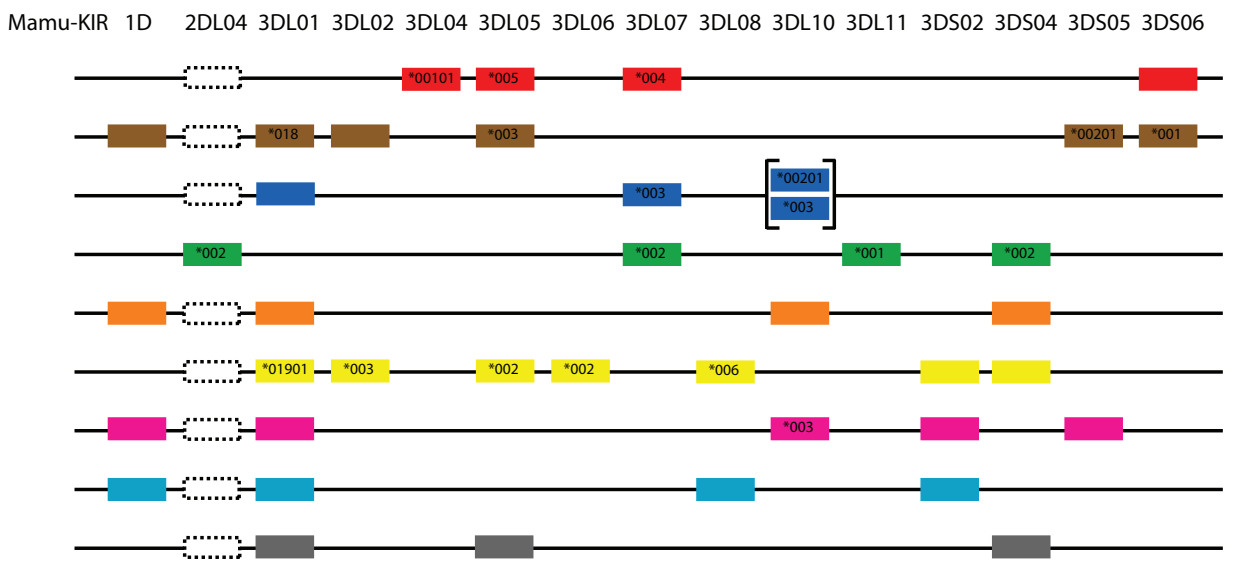

Figure 9 Novel rhesus macaque KIR haplotypes. KIR genes are indicated along the top axis. The identity of the allele is indicated within the schematic boxes if it was determined. Because data were generated from cDNA expression, only expressed KIRs are shown, and the physical map of gene order is arbitrary. Brackets indicate gene duplication. Since Mamu-KIR2DLO4 cannot be amplified by our pyrosequencing amplicon, dotted boxes indicate haplotypes in which a false negative typing for this locus is possible.

$[43,46]$. Preliminary evidence suggests the residues of the Bw4/Bw6 motifs influence KIR/MHC binding in macaques, although the important residues differ from those in humans. Expanding the number of identified KIR/MHC partners and further defining the motifs necessary for macaque KIR/MHC interaction remains essential for the advancement of the field.

\section{Conclusions}

The data and techniques presented here have implications for the study of many diseases using rhesus macaques as a model system and for the KIR field as a whole. The ability to study KIRs in a nonhuman primate model creates an opportunity for significant advancement in our understanding of KIRs in human disease. The novel highthroughput KIR genotyping method we developed has implications for many species, including potential application in humans, and the approach could be adapted for other polymorphic immune loci.

\section{Endnotes}

${ }^{1}$ A note on nomenclature: KIR nomenclature reflects both the domain structure and gene designation of the sequence. For example, an allele named "KIR3DL01"001" denotes a KIR with 3 extracellular immunoglobulin-like domains (3D). The "L" indicates that this allele's protein product has a long, inhibitory cytoplasmic tail, while an "S" would instead indicate that the protein product had a short, activating cytoplasmic tail. The next two digits refer to the gene, in this case KIR3DL01. Finally, the digits after the asterisk, "001" in this example, refer to the specific allele.
${ }^{2}$ Human KIRs are divided into four lineages. Lineage I includes KIR2D genes having a D0 (immunoglobulinlike domain 0) plus D2 configuration such as KIR2DL4 and KIR2DL5; lineage II includes the KIR3DL genes that bind MHC-A and -B epitopes; lineage III includes KIR3D or KIR2D genes with a D1 plus D2 domain configuration that bind MHC-C epitopes, and lineage $\mathrm{V}$ is represented by KIR3DL3. Lineage IV, which included Mamu-KIR3DL01 and Mamu-KIR3DL10, was originally described in Rajalingam, et al. [37]. Further work has instead grouped these KIRs into lineage II and removed lineage IV [47].

\section{Additional material}

Additional file 1: Figure S1. Alignment of all published rhesus macaque lineage II KIR sequences. An alignment was generated from all previously published rhesus macaque KIRs using predicted amino acid sequences. Sequences identified in this publication are highlighted. KIRs are grouped by gene, and a consensus sequence is included for each gene. An asterisk after the accession number indicates this sequence was published multiple times and only one of the accession numbers is given. Table S1. Genbank accession numbers for novel full-length rhesus macaque KIR sequences. Sequences have been assigned official names through the Immuno Polymorphism Database. Table S2. PCR primers used for CDNA-PCR pyrosequencing. Table S3. Comparison of pyrosequencing and cloning results. For each animal, detected KIR alleles are shown, along with their relative frequency detected by pyrosequencing, expressed as a percent of total 454 reads. The column on the right indicates the result of conventional cloning. A plus sign indicates that the detected resolution matches the corresponding pyrosequencing result. If cloning resulted in a different resolution, the corresponding allele name is shown. Table S4. Reproducibility of allele frequency estimates. Pyrosequencing data from four animals are shown. Two independent NK cell isolations were performed per animal, and two independent PCRs were performed per cell preparation, with the exception of 225-07, for which only one cell pellet was available. The 
resulting PCR amplicons were pyrosequenced. The total number of reads is shown for each reaction. KIRs detected are shown, expressed as a percent of total reads. Table S5. GenBank accession numbers for novel partial length rhesus macaque KIR sequences identified by pyrosequencing. Sequences have been assigned sequential, unofficial names. For each sequence, the KIR allele or gene to which it bears greatest similarity is indicated. The Total Ids column indicates the number of distinct animals in which that sequence was observed Abbreviations: u: unique; gc: gene conversion; r: recombination; sv: splice variant.

\section{Acknowledgements}

We thank Roger Wiseman and Simon Lank for advising on technical aspects of this study and for thoughtful comments on this manuscript. We also thank Chris Wright and the staff of the University of Illinois High Throughput Sequencing Center. Finally, we thank Jon Warren and the National Institute of Allergy and Infectious Diseases (NIAID), a component of the National Institutes of Health $(\mathrm{NIH})$, for support. The contents of this manuscript are solely attributed to the authors and do not necessarily represent the officia views of the research sponsors. This publication was made possible in part by grants P51 RR000167 and P51 RR000168 from the National Center for Research Resources (NCRR), a component of the $\mathrm{NIH}$, to the Wisconsin National Primate Research Center and the New England Primate Research Center, respectively along with R24 RR021745 to D.H.O., Al071306 to R.P.J. and AI24258 to PP. Development of KIR pyrosequencing was also supported by the NIH/NIAID Reagent Resource Support Program for AIDS Vaccine Development, Quality Biological, Inc., Gaithersburg, MD. The project was also supported by CHAVI/HVTN Early Career Investigator award, grant number U19 Al 067854-04, to R.K.R.

\section{Author details}

'Department of Pathology and Laboratory Medicine, University of WisconsinMadison, Madison, Wisconsin 53706, USA. ${ }^{2}$ Departments of Structural Biology and Microbiology \& Immunology, Stanford University School of Medicine, Stanford, CA 94305, USA. ${ }^{3}$ Division of Immunology, New England Primate Research Center, Harvard Medical School, Southborough, MA 01772, USA. ${ }^{4}$ Department of Biostatistics and Medical Informatics, University of WisconsinMadison, Madison, Wisconsin 53706, USA. ${ }^{5}$ Wisconsin National Primate Research Center, University of Wisconsin-Madison, Madison, Wisconsin 53706, USA.

\section{Authors' contributions}

AJM was involved in data collection, data analysis and writing the manuscript. LAG and PP performed data analysis and assisted with manuscript writing. RKR performed NK cell sample preparations and was involved in the experimental design. KWB performed statistical analysis of pyrosequencing data. RPJ and DHO provided grant support and were involved in the experimental design. BNB was involved in experimental design, data collection, data analysis and manuscript writing. All authors have read and approve the final manuscript.

Received: 4 April 2011 Accepted: 7 June 2011 Published: 7 June 2011

\section{References}

1. Lanier LL: Evolutionary struggles between NK cells and viruses. Nat Rev Immunol 2008, 8(4):259-268.

2. Gardiner CM: Killer cell immunoglobulin-like receptors on NK cells: the how, where and why. Int I Immunogenet 2008, 35(1):1-8

3. Parham P: MHC class I molecules and KIRs in human history, health and survival. Nat Rev Immunol 2005, 5(3):201-214.

4. Beck JC, Wagner JE, DeFor TE, Brunstein CG, Schleiss MR, Young JA, Weisdorf DH, Cooley S, Miller JS, Verneris MR: Impact of cytomegalovirus (CMV) reactivation after umbilical cord blood transplantation. Biol Blood Marrow Transplant 2010, 16(2):215-222.

5. Miller JS, Cooley S, Parham P, Farag SS, Verneris MR, McQueen KL, Guethlein LA, Trachtenberg EA, Haagenson M, Horowitz MM, et al: Missing KIR ligands are associated with less relapse and increased graft-versus- host disease (GVHD) following unrelated donor allogeneic HCT. Blood 2007, 109(11):5058-5061.

6. Khakoo S, Thio C, Martin M, Brooks C, Gao X, Astemborski J, Cheng J, Goedert J, Vlahov D, Hilgartner M, et al: HLA and NK cell inhibitory receptor genes in resolving hepatitis $C$ virus infection. Science 2004 305(5685):872-874

7. Bonagura VR, Du Z, Ashouri E, Luo L, Hatam LJ, DeVoti JA, Rosenthal DW, Steinberg BM, Abramson AL, Gjertson DW, et al: Activating killer cell immunoglobulin-like receptors 3DS1 and 2DS1 protect against developing the severe form of recurrent respiratory papillomatosis. Hum Immunol 2010, 71(2):212-219.

8. Martin M, Qi Y, Gao X, Yamada E, Martin J, Pereyra F, Colombo S, Brown E, Shupert W, Phair J, et al: Innate partnership of HLA-B and KIR3DL1 subtypes against HIV-1. Nat Genet 2007, 39(6):733-740.

9. Martin M, Gao X, Lee J, Nelson G, Detels R, Goedert J, Buchbinder S, Hoots K, Vlahov D, Trowsdale J, et al: Epistatic interaction between KIR3DS1 and HLA-B delays the progression to AIDS. Nat Genet 2002, 31(4):429-434.

10. Kim S, Poursine-Laurent J, Truscott S, Lybarger L, Song Y, Yang L, French A, Sunwoo J, Lemieux S, Hansen T, et al: Licensing of natural killer cells by host major histocompatibility complex class I molecules. Nature 2005, 436(7051):709-713.

11. Moretta L, Moretta A: Killer immunoglobulin-like receptors. Curr Opin Immunol 2004, 16(5):626-633.

12. Wilson M, Torkar M, Haude A, Milne S, Jones T, Sheer D, Beck S, Trowsdale J: Plasticity in the organization and sequences of human KIR/ ILT gene families. Proc Natl Acad Sci USA 2000, 97(9):4778-4783.

13. Hsu K, Chida S, Geraghty D, Dupont B: The killer cell immunoglobulin-like receptor (KIR) genomic region: gene-order, haplotypes and allelic polymorphism. Immunol Rev 2002, 190:40-52.

14. Witt C, Dewing C, Sayer D, Uhrberg M, Parham P, Christiansen F: Population frequencies and putative haplotypes of the killer cell immunoglobulin-like receptor sequences and evidence for recombination. Transplantation 1999, 68(11):1784-1789.

15. Vilches C, Parham P: KIR: diverse, rapidly evolving receptors of innate and adaptive immunity. Annu Rev Immunol 2002, 20:217-251.

16. Yamada S, Hatta M, Staker BL, Watanabe S, Imai M, Shinya K, SakaiTagawa Y, Ito M, Ozawa M, Watanabe T, et al: Biological and structural characterization of a host-adapting amino acid in influenza virus. PLoS Pathog 2010, 6(8).

17. Shilling HG, Young N, Guethlein LA, Cheng NW, Gardiner CM, Tyan D, Parham P: Genetic control of human NK cell repertoire. J Immunol 2002, 169(1):239-247.

18. Hansen D, D'Ombrain M, Schofield L: The role of leukocytes bearing Natural Killer Complex receptors and Killer Immunoglobulin-like Receptors in the immunology of malaria. Curr Opin Immunol 2007, 19(4):416-423.

19. Alter G, Martin MP, Teigen N, Carr WH, Suscovich TJ, Schneidewind A, Streeck H, Waring M, Meier A, Brander C, et al: Differential natural killer cell-mediated inhibition of HIV-1 replication based on distinct KIR/HLA subtypes. J Exp Med 2007, 204(12):3027-3036.

20. Bontrop RE, Watkins DI: MHC polymorphism: AIDS susceptibility in nonhuman primates. Trends Immunol 2005, 26(4):227-233.

21. O'Connor D, Allen T, Watkins D: Cytotoxic T-lymphocyte escape monitoring in simian immunodeficiency virus vaccine challenge studies. DNA Cell Biol 2002, 21(9):659-664.

22. Loffredo J, Burwitz B, Rakasz E, Spencer S, Stephany J, Vela J, Martin S, Reed J, Piaskowski S, Furlott J, et al: The antiviral efficacy of simian immunodeficiency virus-specific CD8+ T cells is unrelated to epitope specificity and is abrogated by viral escape. J Virol 2007, 81(6):2624-2634

23. Hershberger K, Shyam R, Miura A, Letvin N: Diversity of the killer cell Iglike receptors of rhesus monkeys. J Immunol 2001, 166(7):4380-4390.

24. Grendell RL, Hughes AL, Golos TG: Cloning of rhesus monkey killer-cell Iglike receptors (KIRs) from early pregnancy decidua. Tissue Antigens 2001, 58(5):329-334

25. Sambrook J, Bashirova A, Palmer S, Sims S, Trowsdale J, Abi-Rached L, Parham P, Carrington M, Beck S: Single haplotype analysis demonstrates rapid evolution of the killer immunoglobulin-like receptor (KIR) loci in primates. Genome Res 2005, 15(1):25-35. 
26. Blokhuis JH, van der Wiel MK, Doxiadis GG, Bontrop RE: Evidence for balancing selection acting on KIR2DL4 genotypes in rhesus macaques of Indian origin. Immunogenetics 2009, 61(7):503-512.

27. Kruse $\mathrm{PH}$, Rosner $\mathrm{C}$, Walter L: Characterization of rhesus macaque KIR genotypes and haplotypes. Immunogenetics 2010, 62(5):281-293.

28. Bimber BN, Moreland AJ, Wiseman RW, Hughes AL, O'Connor DH: Complete characterization of killer Ig-like receptor (KIR) haplotypes in Mauritian cynomolgus macaques: novel insights into nonhuman primate KIR gene content and organization. J Immunol 2008, 181(9):6301-6308.

29. Robinson J, Marsh SG: IPD: the Immuno Polymorphism Database. Methods Mol Biol 2007, 409:61-74.

30. Blokhuis JH, van der Wiel MK, Doxiadis GG, Bontrop RE: The mosaic of KIR haplotypes in rhesus macaques. Immunogenetics 2010, 62(5):295-306.

31. Thompson JD, Higgins DG, Gibson TJ: CLUSTAL W: improving the sensitivity of progressive multiple sequence alignment through sequence weighting, position-specific gap penalties and weight matrix choice. Nucleic Acids Res 1994, 22(22):4673-4680.

32. Blokhuis $\mathrm{JH}$, Doxiadis GG, Bontrop RE: A splice site mutation converts an inhibitory killer cell Ig-like receptor into an activating one. $\mathrm{Mol} / \mathrm{mmunol}$ 2009, 46(4):640-648.

33. Li H, Handsaker B, Wysoker A, Fennell T, Ruan J, Homer N, Marth G, Abecasis G, Durbin R: The Sequence Alignment/Map format and SAMtools. Bioinformatics 2009, 25(16):2078-2079.

34. Stajich JE, Block D, Boulez K, Brenner SE, Chervitz SA, Dagdigian C, Fuellen G, Gilbert JG, Korf I, Lapp H, et al: The Bioperl toolkit: Perl modules for the life sciences. Genome Res 2002, 12(10):1611-1618

35. Robinson J, Mistry K, McWilliam H, Lopez R, Marsh SG: IPD-the Immuno Polymorphism Database. Nucleic Acids Res 2010, 38 Database: D863-869.

36. Hershberger $\mathrm{KL}$, Kurian J, Korber BT, Letvin NL: Killer cell immunoglobulinlike receptors (KIR) of the African-origin sabaeus monkey: evidence for recombination events in the evolution of KIR. Eur J Immunol 2005, 35(3):922-935.

37. Rajalingam R, Parham P, Abi-Rached L: Domain shuffling has been the main mechanism forming new hominoid killer cell lg-like receptors. $J$ Immunol 2004, 172(1):356-369.

38. Bashirova AA, Martin MP, McVicar DW, Carrington M: The killer immunoglobulin-like receptor gene cluster: tuning the genome for defense. Annu Rev Genomics Hum Genet 2006, 7:277-300.

39. Kim S, Sunwoo JB, Yang L, Choi T, Song YJ, French AR, Vlahiotis A, Piccirillo JF, Cella M, Colonna M, et al: HLA alleles determine differences in human natural killer cell responsiveness and potency. Proc Natl Acad Sci USA 2008, 105(8):3053-3058.

40. McErlean C, Gonzalez AA, Cunningham R, Meenagh A, Shovlin T, Middleton D: Differential RNA expression of KIR alleles. Immunogenetics 2010, 62(7):431-440.

41. Sharma D, Bastard K, Guethlein LA, Norman PJ, Yawata N, Yawata M, Pando M, Thananchai H, Dong T, Rowland-Jones S, et al: Dimorphic motifs in D0 and D1+D2 domains of killer cell lg-like receptor 3DL1 combine to form receptors with high, moderate, and no avidity for the complex of a peptide derived from HIV and HLA-A*2402. J Immunol 2009, 183(7):4569-4582.

42. Wiseman RW, Karl JA, Bimber BN, O'Leary CE, Lank SM, Tuscher JJ, Detmer AM, Bouffard P, Levenkova N, Turcotte CL, et al: Major histocompatibility complex genotyping with massively parallel pyrosequencing. Nat Med 2009, 15(11):1322-1326.

43. Rosner C, Kruse PH, Hermes M, Otto N, Walter L: Rhesus Macaque Inhibitory and Activating KIR3D Interact with Mamu-A-Encoded Ligands. $\mathrm{J}$ Immunol 2011, 186(4):2156-2163.

44. Budde ML, Lhost JJ, Burwitz BJ, Becker EA, Burns CM, O'Connor SL, Karl JA, Wiseman RW, Bimber BN, Zhang GL, et al: Transcriptionally Abundant Major Histocompatibility Complex Class I Alleles are Fundamental to Non-Human Primate SIV-specific CD8+ T Cell Responses. J Virol 2011.

45. Carrington M, Martin MP, van Bergen J: KIR-HLA intercourse in HIV disease. Trends Microbiol 2008, 16(12):620-627.

46. Colantonio AD, Bimber BN, Neidermyer WJ, Reeves RK, Alter G, Altfeld M, Johnson RP, Carrington M, O'Connor DH, Evans DT: KIR Polymorphisms Modulate Peptide-Dependent Binding to an MHC Class I Ligand with a Bw6 Motif. PLoS Pathog 2011, 7(3):e1001316.

47. Guethlein LA, Older Aguilar AM, Abi-Rached L, Parham P: Evolution of killer cell Ig-like receptor (KIR) genes: definition of an orangutan KIR haplotype reveals expansion of lineage III KIR associated with the emergence of MHC-C. J Immunol 2007, 179(1):491-504

doi:10.1186/1471-2164-12-295

Cite this article as: Moreland et al:: Characterization of killer

immunoglobulin-like receptor genetics and comprehensive genotyping by pyrosequencing in rhesus macaques. BMC Genomics 2011 12:295.

\section{Submit your next manuscript to BioMed Central and take full advantage of:}

- Convenient online submission

- Thorough peer review

- No space constraints or color figure charges

- Immediate publication on acceptance

- Inclusion in PubMed, CAS, Scopus and Google Scholar

- Research which is freely available for redistribution

Submit your manuscript at www.biomedcentral.com/submit
C Biomed Central 